\title{
A Framework for Designing Behavioural Change with the Use of Persuasive Technology
}

\author{
Ana Pintar \\ University of Ljubljana, School of Economics and Business, Slovenia \\ pintar.ana4@gmail.com \\ Jure Erjavec \\ University of Ljubljana, School of Economics and Business, Slovenia \\ jure.erjavec@ef.uni-lj.si
}

\begin{abstract}
Purpose: There is limited integration of behaviour theories and persuasive technology on behavioural change. Previous research calls for employing behaviour change theories to inform their persuasive designs and illustrate how the theories used are linked to the design features. This paper proposes integrating two theoretical models - the compelling system design and the transtheoretical model - to identify which persuasive interventions work best for the user to progress through different stages of behavioural change.
\end{abstract}

Study design/methodology/approach: We performed an in-depth literature review of the transtheoretical model and persuasive system design, followed by an initial framework conceptualization. The initial framework was validated and finalized through in-depth interviews of individuals who have practiced the behaviour change for at least half a year.

Findings: This paper has successfully identified the persuasive system design features and mapped them onto the transtheoretical model stages of change. The stages can be reduced into early and late stages. Consequently, we have developed a framework for designing behavioural change using persuasive technology and validated it through empirical research.

Originality/value: The findings have practical implications for understanding which principles UI and UX and designers should follow when designing products for behavioural change. Moreover, our work provides insights and recommendations for the future design of persuasive interventions that consider the transtheoretical model of change.

Keywords: persuasive technology, behavioural change, compelling system design, transtheoretical model

\section{Introduction}

Smartphones and other mobile devices have become an omnipresent attribute of our daily lives. We use them as a tool that offers new ways of creating, accessing, and sharing information. As a result, numerous applications are being developed that are constantly present in our daily lives. Oinas-Kukkonen and Harjumaa (2009) refer to this phenomenon as technology that is "always-on" and influences people's attitudes and behaviour in one way or another. Technology creators have taken advantage of this and keep developing information technology systems to change users' attitudes and behaviour. Fogg (2002) defined this as a persuasive technology, which has been a well-established trend in the domain of human-computer interaction design and denotes the use of computer technology, focused on the interfaces between people and computers. To that end, a comprehensive framework to design and evaluate systems was developed, termed the persuasive system design (PSD) model, laid out by Oinas, Kukkonen, and Harjumaa (2009). PSD model identifies three potential successful outcomes: 1) voluntary reinforcement, 2) change or shaping attitudes, and 3) change or shaping of behaviours.

Persuasive technology revolves around observing humans' interaction with computers and studying how to design technology that allows people to communicate in a new way. This highlights the need to incorporate the psychology of human behaviour in building persuasive 
technology, so those intervention strategies are designed around psychological and social theories. However, some consideration has been given to applying some of the most promising behavioural change theories to the study of persuasive technologies. Scholars have pointed out the need to employ behaviour change theories to inform their persuasive design and illustrate how the theories are linked to design features. Based on the systematic review (Orji \& Moffatt, 2018), it was identified that approximately 74 per cent of research did not put in use any behaviour theories when designing persuasive systems. However, among employed theories, the transtheoretical model (TTM) was the most frequently used model.

The TTM describes five stages of change through which individuals progress when exhibiting new behaviour: pre-contemplation, contemplation, preparation, action, and maintenance. Each stage is characterized by different intervention strategies that are most effective for the individual to progress and reach the ideal stage of behaviour - the maintenance phase (Ferron \& Massa, 2013). Thus, the purpose behind the TTM is to tailor to specific stages by using activities that support progression through the stages and techniques to build self-efficacy and avoid temptation. Enabling behaviour change to be consistent and longstanding is an open challenge; consequently, combining it with persuasive design opens a new field of opportunities.

Despite the growing body of research in both areas, there is limited integration of behaviour theories and persuasive technology on behavioural change. In this respect, previous research (Orji \& Moffatt, 2018; Ludden \& Hekkert, 2014; Aldenaini, Oyebode, Orji \& Sampalli, 2020) calls for researchers and developers to employ behaviour change theories to inform their persuasive designs. Moreover, it should be illustrated how the theories used are linked to the design features.

Therefore, the purpose of this paper is to propose the integration of two theoretical models the persuasive system design and the transtheoretical model to identify which persuasive interventions work best for the user to progress through different stages of the TTM. The main research question is that we try to answer is how to use the PSD to support individual stages of the TTM. This is further explored by identifying which stages of the TTM benefit the most from the PSD and which PSD elements can be applied in a particular stage of the TTM for designing persuasive technology.

The paper aims at filling the gap between persuasion system design and behavioural theory. The following two sections provide an overview of the transtheoretical model and persuasive system design, followed by an overview of the methodology. Based on theoretical overview and empirical validation, we then develop a framework of design intervention strategies based on the PSD that works best at a particular stage of the TTM and support user progress through the different TTM stages. Finally, the paper's conclusion offers an overview of our research results and discusses limitations and potential further research.

\section{Persuasive system design}

Persuasion is defined as attempting to change attitudes or behaviours or both without using coercion or deception (Fogg, 2002). It denotes influencing one's beliefs towards an idea using different tools or communication approaches to convey feelings or messages. Ideally, persuasion should bring the user to abandon some habits and adopt another. However, there are three possible outcomes: reinforcement, change, and shape (Oinas-kukkonen \& Harjumaa, 2008). Some examples of planned effects might be persuading people to buy things online, reminding people to drink more water, and motivating people to take stretch breaks after extended periods of desk work. Persuasive technology is "an interactive computing system designed to change people's attitudes or behaviour" (Fogg, 2002). In a broader sense, 
persuasive technology incorporates insights from psychology into the design of digital products, such as mobile apps, websites, or wearables. The purpose behind it is to modify or change the individual's behaviour.

Designing persuasive technology is not an easy process to follow; thus, it requires numerous techniques and strategies to succeed. In his book, Fogg (2002) introduced the functional triad as a framework that highlights the roles of computing products and makes it easier to design persuasive systems. Firstly, computers can take the role of social actors by creating relationships, rewarding users with positive feedback, and providing social support. Secondly, computers as a medium provide an experience that motivates and helps people practice behaviour. Lastly, computers can serve as tools by increasing capability, making activities easier to do, leading people through the process, and performing calculations that motivate the user towards achieving a target behaviour.

The critical framework disadvantage is the limited possibility of strategies directly applied when designing persuasive systems; therefore, Persuasive System Design (PSD) was proposed (Oinas-Kukkonen, 2009). It is comprised out of three core elements: 7 postulates behind persuasive systems, 3 ways to analyse the context, and 28 design principles. Thus, PSD consists of three key steps; firstly, to understand the assumptions behind persuasive systems, analyse the context, and draw out design principles.

The PSD's postulates are standards used to meet non-functional software requirements. The context can either be an intent, which includes the persuader and the change type, an event, which analyses the context of use, the user context, and the technology context; or a strategy, which includes the need to identify the route and message. The context itself describes the situation within which something happens, and the issue must be situated in the context before we can understand it. Hence, the persuasion context is used to consider conditions where persuasion takes place, and it is a crucial step first to understand and later promote behaviour change. The final stage of the PSD framework is divided into four categories of design principles: primary task support, dialogue support, system credibility, and social support. Various techniques for designing actual features, content, and functionality are defined in each category, so the artefact ends up being more persuasive. The purpose is to understand what strategies have the most significant influence on the user's behaviour change.

PSD framework has been applied in many areas of human-computer interaction design. In general, persuasive technology designers aim to address specific challenges within certain areas, for example, obesity, alcoholism, internet addiction, environmental issues, and learning disabilities. To illustrate that, healthcare software applications might be developed either to encourage people towards healthy behaviour (Consolvo, Landay \& Mcdonald, 2009; Jafarinaimi, Forlizzi, Hurst \& Zimmerman, 2005; Lin, Mamykina, Lindtner, Delajoux \& Strub, 2006; Maitland et al., 2006; Dantzig, Geleijnse \& Halteren, 2013) or to manage and prevent diseases and health conditions (Intille, 2003; Kraft, Drozd \& Olsen, 2009). Furthermore, persuasive systems within environmental conservation can provide effective solutions aiming to support sustainable energy and water consumption behaviour (Kappel \& Grechenig, 2009; Kuznetsov \& Paulos, 2010; Elsmore, Wilson, Jones \& Eslambolchilar, 2010). In the same way, the PSD model can be applied in green mobility initiatives and smart city solutions (Jylhä, Nurmi, Sirén, Hemminki \& Jacucci, 2013; Meloni \& di Teulada, 2015). Persuasive systems can also be used in education in assisting the learning process as a support for pedagogical activities or as a tool to persuade users in general education activities (Dewi, Widyasari, Nugroho \& Permanasari 2019). Despite many applications where the PSD has been implemented, it has received some critiques (Wiafe, Alhammad, Nakata \& Gulliver, 2012; Corbett, 2013) for being 
too general and not providing precise guidelines for engineers and designers on how actually to implement the described principles.

\section{The transtheoretical model of behaviour change}

Human behaviour is any observable physical or verbal activity made by an individual or group. On the one hand, it is driven by factors, such as thoughts, feelings, beliefs, values, and other psychological traits unique to each individual, and on the other hand by many different factors such as environment, social norms, religion, attitude, weather, and others (Lazzeri, 2014). A crucial aspect of human behaviour is a habit, which denotes the routine of regularly repeated behaviour that usually occurs subconsciously, a formula our brain automatically follows (Duhigg, 2014). Habits influence how people work, eat, play, live, spend, and communicate; hence, forming good habits is crucial for living our lives to the fullest. However, bad habits such as smoking or binge eating indeed exist. Even though people are aware of them, and know precisely what strategies they should use to change their habitual behaviour, they still struggle.

To study human behaviour and, more so, to understand its complexity and thus, to help people change destructive behavioural patterns, many theories have emerged. However, research has shown that much persuasive technology is developed and designed without any support of behaviour theories. Nevertheless, when behaviour theories are employed, the transtheoretical model of behaviour change (TTM) appeared to be the most effective of all (Orji \& Moffatt, 2018).

TTM consist of the following constructs: stages of change, processes of change, decisional balance, self-efficacy, and temptation. Processes of change include activities that individuals use to modify their thoughts, feelings, and behaviour to progress through the stages. In addition, they represent essential guides for designing intervention strategies. The ones that received the most empirical support to date are consciousness-raising, dramatic relief, self-reevaluation, environmental re-evaluation, self-liberation, social liberation, counterconditioning, stimulus control, contingency management, helping relationships. Different processes of change need to be emphasized at various stages of change. The early stages should focus on cognitive, evaluative, and affective processes, while the later stages that are more action-oriented should focus on contingencies, commitments, conditioning, and environmental control (Glanz, Rimer \& Viswanath, 2015). According to Prochaska and Velicer (1997), the transtheoretical model involves progress through six stages of change: pre-contemplation, contemplation, preparation, action, maintenance, and termination.

In the pre-contemplation phase, there is no intention to change the behaviour within the next 6 months. On the other hand, an individual recognizes problematic behaviour in the contemplation phase and intends to change it within the next 6 months. The preparation phase includes planning to change, already taking small actions. An action phase follows, where an individual performs consistent behavioural change for less than 6 months. Finally, the maintenance phase required successful maintenance of behavioural change for 6 months, whereas reaching the termination phase implies that an individual has no temptation to relapse. The six stages of the TTM represent individual readiness or propensity to engage in particular an activity that might lead towards the desired behaviour. The concept of the stage is crucial as it reflects a temporal dimension in which change unfolds coupled with a set of tasks required for progressing to the next stage.

Notwithstanding the different time individuals spend in each stage, the tasks to be done to progress to the following stages are equal. Therefore, the stages serve as an essential step for understanding specific behavioural patterns. Furthermore, targeted behaviour is achieved by moving through each stage of behaviour change; hence, skipping stages is not an option as it 
might result in a high risk for relapse (Brinthaupt \& Richard P. Lipka, 1994). Furthermore, the TTM addresses behavioural change as a dynamic and sequential process and nonlinear progression. Therefore, the user can also regress from later stages to earlier ones or remain in the same stage for an extended period of time.

Although the TTM is a sequential process, nonlinear progress across phases is also a possible scenario. For example, an individual can return from the later stages to an earlier stage or remain in the same stage without moving forward for an extended period of time (Ferron \& Massa, 2013). Furthermore, behaviour change is a complex and dynamic process characterized by different motivational levels; thus, adopting an adaptive, personalized approach might be the most effective way to encourage users towards healthier behaviour change.

TTM describes one's readiness to act on a new desired behaviour (Norcross \& Goldfried, 2005). Defined as a model of intentional change, it functions on the premise that people do not change behaviour decisively and quickly, but slowly and continuously through a cyclical process. Behavioural change was often defined as an event, such as starting exercising, though change occurs over time as progress through specific stages as individuals struggle to remove problematic behaviour. Hence, it is conceptualized as a longitudinal and dynamic process across different stages (Prochaska \& Velicer, 1997).

\section{Methodology}

To meet the paper's purpose and answer the research questions, we first conducted a theoretical overview presented in the previous two sections, which has provided a deep insight into the study setup. Then, by studying the TTM, we have identified individual stages that could benefit the most from applying the PSD elements. Based on the characteristics of each TTM stage, we have identified the need that represents a guideline for designing an intervention. Furthermore, we have used the PSD design of the system feature part of the model to link individual feature requirements to a particular TTM stage.

To validate and further refine the conceptual framework, we conducted in-depth interviews. The criteria for selecting respondents were 1) the maintenance stage of the TTM model and 2) behavioural change in the health domain. Respondents have had to practice the behaviour for more than half a year, and the change had to relate to either physical activity, diet change, or meditation. We have interviewed five people, out of these two males and three females. The men belong to the age group of 30-40, while the women were from the age group of 20-30. The design of the questionnaires was based on findings from the theoretical overview. The questions were designed according to the qualitative research guidelines provided by Jacob and Furgerson (2012) and Turner (2010). The main questions were coupled with probing questions and examples. The purpose was to validate if stage-matched interventions based on the PSD are helpful in the TTM stages. The questions aimed to validate all the stage interventions; however, the focus was on the maintenance stage. The interview contained the following 11 main questions: (1) Tell me about your behavioural change.; (2) How did your decision-making process of starting behaviour change look like? (3) What emotions and feelings did you experience at the beginning? (4) How did you prepare yourself for behavioural change? (5) How did your action stage look like? (6) Tell me about temptations. (7) How do you perform the desired behaviour change, and how do you keep going? (8) What emotions and feelings do you experience now in the maintenance stage? (9) What role does tracking have in maintaining your behaviour? (10) What role does social influence have on maintaining your behaviour? (11) What support can technology offer you to keep maintaining change.

Lastly, we have translated the outcomes from interviews to intervention strategies, combined them with concepts derived from the theoretical overview, and as a result, designed a 
framework that will provide insights and recommendations for future persuasive design strategies. The research process is shown in Figure 1.

Literature
review

Figure 1: Research process

\section{Framework development and validation}

The purpose of the literature review was to present and analyse both models in detail and to identify the specific areas where the models complement each other. In this section, we use the system features part of the PSD model and link an individual feature requirement to a particular stage of the TTM. Firstly, we identify the characteristic of all the TTM stages of change. Secondly, we build upon the already established TTM construct - the process of change-to determine each stage's need and intervention requirements. Lastly, we map the PSD system features that best match the needs of individual stages and serve as a guideline for designing stage-matched interventions.

Designing stage-matched interventions is a crucial aspect to consider when designing with the goal of behavioural change. Despite the process of change generally incorporating core ideas of how interventions should be prepared and which stage they should be matched to, they have some downsides. One of them is not putting enough emphasis on computer-based interactive interventions that emerged with new technology advancements. Processes of change have focused mainly on between-person interaction. Still, the advancement of technology brought new opportunities to explore behaviour change by collecting individual-level data, using mobile devices, wearable gadgets, mapping the digital footprint of online activities, and continuous monitoring (Naslund et al., 2017).

In the pre-contemplation stage, the user feels unmotivated, resistant to change, and lacks knowledge about the consequences of not changing their behaviour. Therefore, there is a need for interventions to focus on education, motivation, and increasing awareness at this stage. In other words, interventions should focus on revealing the users' behaviours to have a clear understanding of their current behavioural patterns and observe their behaviour. In the context of change processes, the need is addressed by stimulus control, conscious raising, and dramatic relief. The characteristics describing the contemplation stage are ambivalence, chronic contemplation, and behavioural procrastination. People at this stage are aware of the consequences, understand the benefits of changing, and have the intention to change but did not commit yet. To that end, the need for interventions at this stage is to raise awareness and help people understand why their behaviour is problematic.

Furthermore, interventions are more effective if they raise emotions. The preparation stage is characterized by people having the intention to perform behaviour change; hence they have 
already developed a plan of action and started taking small actions such as buying a book or joining a program. The need for this stage's interventions is to reengineer the environment so that performing a behaviour is as simple as possible. The aim is to engage people in the change of behaviour, perhaps by recommending or suggesting different types of actions and to make people learn about the dynamics of the phenomenon. In the action stage, individuals feel motivated, yet there is the highest risk for relapse in this stage. This highlights the need for intervention to be protected and support-oriented, meaning they should protect users from risky contexts and help to avoid problematic behaviour.

Furthermore, there is also a need to provide support for possible undesired behaviours. For example, an individual in the maintenance stage feels confident and is less tempted to relapse. The core need in this stage is to reinforce the desired behaviour and maintain change to make it sustainable. Additionally, it is beneficial to assess the conditions under which one can relapse and develop alternatives to help them not fall into the relapse phase.

Sometimes it is difficult to draw the line between the PSD elements that should be applied at specific stages since the early stages and the late stages have similar needs and characteristics. For example, action and maintenance are both focused on social influence; hence, some elements from social support might also be a good fit for designing interventions in the final stage. When designing systems, the ground rule must follow the need of the early stages to be understanding-focused, while the later stages are action-focused. We have marked some PSD system features as "universal", which means they should be used throughout the entire system, regardless of the stage the user is at. These principles are trustworthiness, expertise, surface credibility, authority, real-world feel, third-party endorsements, verifiability (system credibility), and liking (dialogue support). The need for the system to be trustworthy and credible for the user is the primary criteria designers should keep in mind. These are not niceto-have features but must-have features. The same goes with liking, which aims at a system being visually attractive.

The interview results provided an important insight into the stages of change and their characteristics and can be summed up into 8 main conclusions: (1) the need to apply for social support already in (pre)contemplation stage; (2) the social circle is not that social, it is a friend/mentor circle; (3) an experiment as a starter boost; (4) simulation, rehearsal, and normative influence as the most significant potential; (5) interventions for addressing negative emotions; (6) technology as a (psycho)therapist; (7) making it fun; (8) the need for more earlystage interventions.

When analysing results, many PSD elements that we initially mapped at specific stages apply to other stages as well. Moreover, some of the respondents remarked they did not exactly feel the particular changes of change. To put it simply, they contemplated for a long time, but after getting the right trigger and really deciding to change, they quickly progressed into the action stage. Thus, even though the respondents subconsciously went through the stages of change, it appeared much more effective for the PSD elements not to be strictly linked to one stage but instead expanded over more of them. Based on this finding, we concluded that when mapping the PSD onto the TTM, the stages of change can be reduced into the early stages that focus on understanding, learning, and motivation, and the later stages that concentrate on resisting temptations, performing the desired behaviour, and maintaining it. According to the terminology used by Laurillau, Calvary, Foulonneau, and Villain (2006), the early stages are termed understanding-oriented while the later ones are action-oriented. Their argumentation goes hand in hand with our findings. Hence, we propose a framework that follows this view. Thus, the main criteria for mapping the PSD elements onto the TTM is that the early stages system feature should be focused on providing education, comprehension, and motivation. In 
contrast, the later stages include system features concentrated around reinforcing the desired behaviour. Figure 2 provides an overview of a new proposed framework.

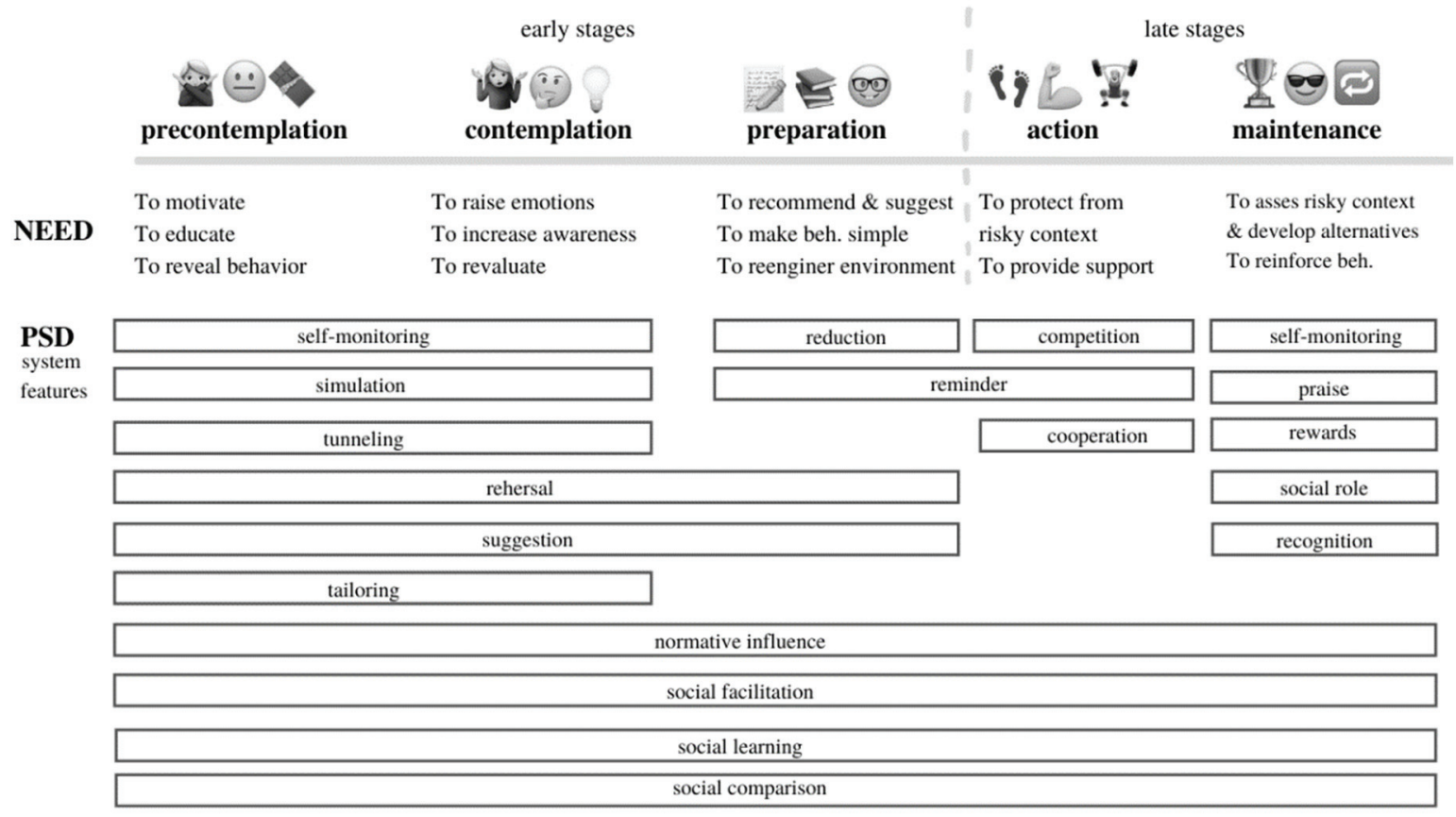

Figure 2: Framework for designing behavioural change with the use of persuasive technology

In general, the early stages have more proposed features than the later stage, as respondents initially spent more time and had more difficulties compared to the later stage. This brings us to a conclusion; since (pre)contemplation stages sometimes take years, it might be worth considering how to shorten this phase by supporting it with the PSD.

\section{Conclusion}

Previous research has shown that the persuasive technology field lacks the integration of behavioural theory. Aiming to fill this gap, we have successfully identified the PSD system features and mapped them onto the TTM stages of change. Based on the literature review and verified by in-depth interviews, we were able to design a framework for creating behavioural change using persuasive technology.

These findings have practical implications for understanding which principles UI and UX and designers should follow when designing products for behavioural change. Moreover, our work provides insights and recommendations for the future design of persuasive interventions that consider the transtheoretical model of change. The framework we have proposed could serve interface designers to firstly identify in which stage of change their potential users are at, and then accordingly match the intervention to create software applications persuasively and make the users change their behaviour. There are 4 concrete suggestions we would give the designers and business analysts developing products for behavioural change: (1) pick a target group and conduct user research to find out in which stage of change the users are; (2) try to understand users' emotions; (3) link the PSD features according to the TTM stage; (4) give extra attention to simulation, rehearsal, and normative influence. What can be learned from this paper is that developing software applications does not only require technical knowledge, but a deep understanding of human psychology, behavioural theories, or, more specifically, how human minds work and how decisions are made. According to this paper, it is clear that software feature requirements should be thoughtfully mapped on one's stage of change. 
However, there are still plenty of opportunities to be explored to bridge the gap between product development and behavioural science. Firstly, further research could be conducted with an increased sample size and use questionnaires with Likert scales to analyse users' attitudes toward specific features. Secondly, these findings highlight the need to investigate further how we could better support the early stages of the TTM. Perhaps this could be done by conducting experiments and longitudinal studies to evaluate the effectiveness level of the stage-matched interventions at different time points during behavioural change. Thirdly, it would be interesting to build an actual mobile application, a prototype, that would incorporate stage-matched intervention and give it to users to test it. That way, it would be possible to objectively measure the effectiveness of system features by data collection via Google Analytics, Hotjar, or some other data analytics tool. Lastly, since interventions vary from context to context, it would be interesting to examine further which strategies work best under a particular context. Finally, other studies could also assess whether stage-matched interventions are clear enough for UI/UX designers to translate them into actual design components.

\section{References}

Aldenaini, N., Oyebode, O., Orji, R., \& Sampalli, S. (2020). Mobile Phone-Based Persuasive Technology for Physical Activity and Sedentary Behavior: A Systematic Review. Frontiers in Computer Science, 2(1), 73 79.

Brinthaupt, T. M., \& Richard P. Lipka, E. (1994). Changing the Self: Philosophies, techniques, and experiences. New York: State University of New York Press.

Consolvo, S., Landay, J. A. \& Mcdonald, D. W. (2009). Designing for Behavior Change in Everyday Life. Computer, 42(6), 86-89.

Corbett, J. (2013). Designing and Using Carbon Management Systems to Promote Ecologically Responsible Behaviors. Journal of the Association for Information Systems, 14(7), 2.

Dantzig, S., Geleijnse, G., \& Halteren, A. (2013). Toward a persuasive mobile application to reduce sedentary behavior. Personal and ubiquitous computing, 17(6), 1237-1246.

Dewi, Y., Widyasari, L., Nugroho, L. E., \& Permanasari, A. E. (2019). Persuasive technology for enhanced learning behavior in higher education. International Journal of Educational Technology in Higher Education, 16(1), 1-16.

Duhigg, C. (2014). The Power of Habit: Why We Do What We Do in Life and Business. Random House Trade Paperbacks.

Elsmore, C., Wilson, M. L., Jones, M., \& Eslambolchilar, P. (2010). Neighbourhood Watch - Community Based Energy Visualisation For The Home. In Proceedings of the First International Workshop on Nudge \& Influence Through Mobile Device. (pp. 23-26). Swansea: Swansea University

Ferron, M., \& Massa, P. (2013). Transtheoretical model for designing technologies supporting an active lifestyle. In Proceedings of the Biannual Conference of the Italian Chapter of SIGCHI (pp. 1-8).

Fogg, B. J. (2002). Persuasive technology: using computers to change what we think and do. Burlington: Morgan Kaufmann.

Glanz, K., Rimer, B. K. \& Viswanath, K. (2015). Health behavior: Theory, Research and Practice. Hoboken: John Wiley \& Sons.

Intille, S. S. (2003). Ubiquitous Computing Technology for Just-in-Time Motivation of Behavior Change. Studies in Health Technology and Informatics, 107(2), 1434-1437.

Jacob, S. A. \& Furgerson, S. P. (2012). The qualitative report writing interview protocols and conducting interviews: Tips for students new to the field of qualitative research. The Qualitative Report, 17(42), 1-10.

Jafarinaimi, N., Forlizzi, J., Hurst, A. \& Zimmerman, J. (2005). Breakaway: an ambient display designed to change human behavior. In CHI'05 extended abstracts on Human factors in computing systems (pp. 1945-1948).

Jylhä, A., Nurmi, P., Sirén, M., Hemminki, S. \& Jacucci, G. (2013). Matkahupi: a persuasive mobile application for sustainable mobility. In Proceedings of the 2013 ACM Conference on Pervasive and Ubiquitous Computing Adjunct Publication (pp. 227-230). New York: Association for Computing Machinery.

Kappel, K. \& Grechenig, T. (2009). Show me: water consumption at a glance to promote water conservation in the shower. In Proceedings of the 4th International Conference on Persuasive Technology (pp. 1-7). New York: Association for Computing Machinery.

Kraft, P., Drozd, F. \& Olsen, E. (2009). Digital therapy: Addressing willpower as part of the cognitive-affective processing system in the service of habit change. In International Conference on Persuasive Technology (pp. 177-188). Berlin: Springer. 
Kuznetsov, S. \& Paulos, E. (2010). UpStream: motivating water conservation with low-cost water flow sensing and persuasive displays. In Proceedings of the SIGCHI Conference on Human Factors in Computing Systems (pp. 1851-1861). New York: Association for Computing Machinery.

Laurillau, Y., Calvary, G., Foulonneau, A. \& Villain, E. (2016). SEPIA, a support for engineering persuasive interactive applications: properties and functions. In Proceedings of the 8th ACM SIGCHI Symposium on Engineering Interactive Computing Systems (pp. 217-228). New York: Association for Computing Machinery.

Lazzeri, F. (2014). On defining behavior: some notes. Behavior and Philosophy, 42, 65-82.

Lin, J. J., Mamykina, L., Lindtner, S., Delajoux, G. \& Strub, H. B. (2006). Fish'n'Steps: Encouraging physical activity with an interactive computer game. In International conference on ubiquitous computing (pp. 261278). Berlin: Springer.

Ludden, G. D. S. \& Hekkert, P. (2014). Design for healthy behavior design interventions and stages of change. In Proceedings of the Colors of Care: The 9th International Conference on Design \& Emotion. Ediciones Uniandes (pp. 482-488). Bogotá: Cali \& Medellin.

Maitland, J., Sherwood, S., Barkhuus, L., Anderson, I., Hall, M., Brown, B., Chalmers, M. \& Muller, H. (2006). Increasing the awareness of daily activity levels with pervasive computing. In 2006 Pervasive Health Conference and Workshops (pp. 1-9). New York: IEEE.

Meloni, I. \& di Teulada, B. S. (2015). I-Pet Individual Persuasive Eco-travel Technology: A tool for VTBC program implementation. Transportation Research Procedia, 11, 422-433.

Naslund, J. A., Aschbrenner, K. A., Kim, S. J., Mchugo, G. J., Bartels, S. J. \& Marsch, L. A. (2017). Health Behavior Models for Informing Digital Technology Interventions for Individuals With Mental Illness. Psychiatric rehabilitation journal, 40(3), 325.

Norcross, J. C. \& Goldfried, M. R. (2005). Handbook of psychotherapy integration. Oxford: Oxford University Press.

Oinas-Kukkonen, H. \& Harjumaa, M. (2008). A systematic framework for designing and evaluating persuasive systems. In International conference on persuasive technology (pp. 164-176). Berlin: Springer.

Oinas-Kukkonen, H. \& Harjumaa, M. (2009). Persuasive systems design: Key issues, process model, and system features. Communications of the Association for Information Systems, 24(1), 485-500.

Orji, R. \& Moffatt, K. (2018). Persuasive technology for health and wellness: State-of-the-art and emerging trends. Health informatics journal, 24(1), 66-91.

Prochaska, J. O., \& Velicer, W. F. (1997). The transtheoretical model of health behavior change. American Journal of Health Promotion, 12(1), 38-48.

Turner, D. W. (2010). Qualitative interview design: A practical guide for novice investigators. Qualitative Report, 15(3), 754-760.

Wiafe, I., Alhammad, M. M., Nakata, K. \& Gulliver, S. R. (2012). Analyzing the persuasion context of the persuasive systems design model with the 3D-RAB model. In International Conference on Persuasive Technology (pp. 193-202). Berlin: Springer. 